\title{
A simulation study of homogeneous ice nucleation in supercooled salty water
}

\author{
Guiomar D. Soria ${ }^{\dagger}$, Jorge R. Espinosa ${ }^{\dagger}$, Jorge Ramirez ${ }^{\S}$,Chantal Valeriani ${ }^{\dagger}, \ddagger$, Carlos Vega ${ }^{\dagger}$ and Eduardo Sanz \\ ${ }^{\dagger}$ Departamento de Quimica Fisica I, \\ Facultad de Ciencias Quimicas, \\ Universidad Complutense de Madrid, 28040 Madrid, Spain. \\ ${ }^{\ddagger}$ Departamento de Fisica Aplicada I, \\ Facultad de Ciencias Fisicas, \\ Universidad Complutense de Madrid, 28040 Madrid, Spain. \\ $\S$ Departamento de Ingenieria Quimica Industrial y Medio Ambiente, \\ Escuela Tecnica Superior de Ingenieros Industriales, \\ Universidad Politecnica de Madrid, 28006 Madrid, Spain
}

(Dated: July 4, 2018)

\begin{abstract}
We use computer simulations to investigate the effect of salt on homogeneous ice nucleation. The melting point of the employed solution model was obtained both by direct coexistence simulations and by thermodynamic integration from previous calculations of the water chemical potential. Using a Seeding approach, in which we simulate ice seeds embedded in a supercooled aqueous solution, we compute the nucleation rate as a function of temperature for a $1.85 \mathrm{NaCl}$ mole per water kilogram solution at 1 bar. To improve the accuracy and reliability of our calculations we combine Seeding with the direct computation of the ice-solution interfacial free energy at coexistence using the Mold Integration method. We compare the results with previous simulation work on pure water to understand the effect caused by the solute. The model captures the experimental trend that the nucleation rate at a given supercooling decreases when adding salt. Despite the fact that the thermodynamic driving force for ice nucleation is higher for salty water for a given supercooling, the nucleation rate slows down with salt due to a significant increase of the ice-fluid interfacial free energy. The salty water model predicts an ice nucleation rate that is in good agreement with experimental measurements, bringing confidence in the predictive ability of the model.
\end{abstract}

\section{INTRODUCTION}

The formation of ice from supercooled water is arguably the most important freezing transition on Earth. Such transition has important consequences in geology 1], food industry 2, 3], or cryopreservation [4]. Most water on Earth contains dissolved salt. Therefore, understanding freezing in salty water is of out-most importance. In this work we focus on homogeneous nucleation, the case in which ice starts growing in the bulk supercooled liquid. Salt hinders ice formation both by decreasing its melting temperature, $T_{m}$, and by increasing the supercooling with respect to $T_{m}$ required to observe freezing [5]. We aim at understanding the latter effect in this paper.

The first step of the freezing transition is the nucleation of a critical crystal cluster -one that has equal chances to melt or irreversibly grow [ $[6]$. The number of such critical clusters appearing per unit time and volume is the nucleation rate, $J$. There are several experimental works where $J$ has been measured for ice in salty water [5, 7]. While the homogeneous nucleation rate can be experimentally measured in careful experiments to avoid the presence of impurities, other relevant nucleation parameters such as the size or the structure of the cluster, or the ice-liquid interfacial free energy are difficult to obtain in experiments [8].

Molecular simulations are an excellent complement to experimental studies of the freezing transition because they have access to detailed information at the molecu- lar scale [9]. There are many simulation studies of ice nucleation in pure water (e.g. 9-17|), and salt precipitation in supersaturated solutions [18 21], but not much work has been devoted to study freezing of salty water 22 24]. In the few existing studies, neither nucleation rates nor ice-solution interfacial free energies have been calculated.

Like salt, pressure is known to slow down ice nucleation [5, 25]. After having studied the effect of applying high pressure on homogeneous ice nucleation [26], we performed a comparative study of the effects of pressure and salt where we concluded that both factors hinder ice nucleation by increasing the ice-liquid interfacial free energy [27]. In this paper we focus the discussion on the effect of salt alone and provide details of our study of homogeneous ice nucleation in salty solutions that were not given in Ref. [27].

We study ice nucleation in a $1.85 \mathrm{NaCl}$ mole per water kilogram solution $(1.85 \mathrm{~m})$ aqueous solution using a model that combines TIP4P/2005 water 28] and the Joung Cheetham/SPC/E NaCl [29] model [30].

We attempt to rationalise the nucleation rate by examining the variables upon which it depends according to Classical Nucleation Theory [6, 31, 32]. First, we determine the melting temperature of ice in a 1.85 $\mathrm{m} \mathrm{NaCl}$ solution at normal pressure via two different routes, one based on direct coexistence simulations between both phases 33] and another based on calculations of the chemical potential of the bulk phases separately 30, 34]. Using thermodynamic integration from 
the melting point 35] we compute the chemical potential difference between ice and water in solution. We then obtain via seeded simulations the size of critical ice clusters for three different temperatures [36]. By simulating a critical cluster we estimate the frequency with which molecules attach to it [37]. To improve the reliability of our calculations we also compute the interfacial free energy at coexistence (a thermodynamic parameter that cannot be reliably measured experimentally) with the Mold Integration method [38, 39]. Combining the simulation results with Classical Nucleation Theory (the seeding method [36]) we estimate the nucleation rate and the interfacial free energy for a wide supercooling range. To rationalise the effect of salt on ice nucleation we compare these results with those previously obtained for homogeneous ice nucleation in pure water [17, 40].

\section{MODEL}

We use the TIP4P/2005 model for water 28]. For $\mathrm{NaCl}$ we use the Joung Cheetham model parametrised for SPC/E water [29], although in this work we combine it with TIP4P/2005 water as in Ref. [30]. Notice that it would not be a good idea to use SPC/E water as solvent for this study because its melting point for pure water is about $215 \mathrm{~K}$ (too far from from the experimental value) and besides the kinetics for the supercooling considered in this work (i.e up to $40 \mathrm{~K}$ ) would be terribly slow for this model. The ion-water cross interaction follows the Lorentz-Berthelot combination rules [41, 42] for the Lennard-Jones part and the Coulomb law for the electrostatic part. The solubility limit for this brine solution force field at 1 bar and $298 \mathrm{~K}$ is $3.5 \mathrm{~m}(\mathrm{NaCl}$ mol per $\mathrm{H}_{2} \mathrm{O} \mathrm{kg}$ ) [30], somewhat smaller than the experimental value of $6.15 \mathrm{~m} \mathrm{[43]}$.

\section{SIMULATION DETAILS}

All runs are performed at constant pressure of $p=$ 1 bar and $\mathrm{NaCl}$ concentration of $1.85 \mathrm{~m}$.

All our simulations are run with the Molecular Dynamics (MD) GROMACS package [4]. We use a ParrinelloRahman barostat 45] with a relaxation time of $0.5 \mathrm{ps}$ to fix the pressure. To keep the temperature constant we employ a velocity-rescale thermostat [46] with a relaxation time of $0.5 \mathrm{ps}$. The time step for the Verlet integration of the equations of motion is $2 \mathrm{fs}$. We use Particle Mesh Ewald Summations [47] to deal with electrostatic interactions. The cut-off radius for both dispersive interactions and the real part of electrostatic interactions is 9 $\AA$. The LINCS algorithm is used to fix the geometry of the water molecules [48, 49].

\section{METHODS}

To obtain the melting temperature for the selected water-ion force field we use two different approaches. On the one hand we use the so-called direct coexistence method [33]. Such method consists in simulating the solid in contact with the liquid at several temperatures [22, 23]. Below the melting temperature, the solid grows at the expense of the fluid phase, and vice versa. In this way the melting temperature can be enclosed within a certain range. The method has been used in the past by some of us to obtain the melting temperature of pure water [50], hard spheres [51, 52] or sodium chloride [53]. On the other hand, we perform thermodynamic integration 35] from previous calculations of the chemical potential of the solvent and the solute [30, 34] and from the melting point of pure water [54] in order to find the melting temperature as that for which the chemical potential of water in ice and in solution coincide. The calculation of the chemical potential of the components of a solution is a difficult task that has recently received great attention in the context of the computation of crystal solubilities 55 60.

Ice nucleation at moderate supercooling is beyond the time scale of standard Molecular Dynamics simulations. Special techniques are needed to promote the nucleation (rare) event. In this work we use the seeding method, which has recently been carefully validated by us [36]. The seeding technique consists in inserting an ice cluster in the supercooled fluid (salty water) and then simulating the resulting equilibrated configuration at several temperatures to obtain the temperature at which the inserted cluster reaches a critical size. Such information, combined with Classical Nucleation Theory [6, 31, 32] provides estimates of important quantities for the nucleation process such as the ice-liquid interfacial free energy 61, 62], the height of the nucleation free energy barrier 63, 64] and the nucleation rate [17, 26, 40, 65 67]. The latter requires launching MD trajectories from the critical cluster in order to get the kinetic prefactor.

To compute the ice-solution interfacial free energy at coexistence (for a flat interface) we use the Mold Integration method, that has been developed by some of us [38] and employed to study the crystal-fluid interface for $\mathrm{NaCl}$ and water [26, 39, 66]. The method consists in gradually inducing the formation of a crystal slab in the fluid at coexistence conditions with the aid of a mold of potential energy wells placed at the lattice sites of a crystal plane 38. The work needed to form such slab can be obtained by thermodynamic integration and is directly related to the crystal-fluid interfacial free energy [38].

We refer the reader to Refs. [51] (direct coexistence), [36] (seeding) and [38] (Mold Integration) for a detailed description of the employed methods. Nevertheless, in the following section we briefly explain these methods as we present and discuss the results. 


\section{RESULTS}

\section{A. Melting temperature}

To start with, we compute the melting temperature, $T_{m}$ of ice in contact with the $\mathrm{NaCl}$ solution at the chosen $\mathrm{NaCl}$ concentration $(1.85 \mathrm{~m})$. We perform such calculation with two different methods: direct coexistence and and thermodynamic integration.

\section{Direct coexistence}

In the direct coexistence method an ice and a 1.85 $\mathrm{m} \mathrm{NaCl}$ solution slab are put at contact as shown in Fig. 1 (a). Simulations are carried out in the anisotropic isothermic-isobaric NpT ensemble [52]. When the imposed temperature is below $T_{m}$ the ice slab grows, and vice versa. In order to know whether the ice slab grows or melts we monitor $N$, the number of molecules in the ice phase (see appendix $\mathrm{A}$ ). In Fig. 1(b) we plot $N$ versus time for different temperatures. At $238 \mathrm{~K}$ the number of ice-like particles grows whereas it decreases at $240 \mathrm{~K}: T_{m}$ is thus $239 \pm 1 K$.

In order to use an unambiguous criterion to determine $T_{m}$ we compute the slope of a linear fit to each $N(t)$ trajectory in Fig. 1(b). If the slope is positive, ice is growing and viceversa. Therefore, the temperature at which the slope is zero corresponds to $T_{m}$. The slope (in molecule/ns units) as a function of temperature is shown in the inset of Fig. 1(b). As announced, the slope becomes 0 at $T_{m}=239 K$.

When estimating $T_{m}$ with the direct coexistence method, the salt concentration may change as water molecules are added/removed to/from the solution due the ice melt/growth. This is an undesirable finite size effect because we are interested in obtaining $T_{m}$ for a specific value of the concentration $(1.85 \mathrm{~m})$. To alleviate such finite size effect we took three measures: (i) use a large system size (23100 water molecules, of which 4050 were in the ice phase $+638 \mathrm{NaCl}$ ) (ii) use a solution slab much larger than the ice slab (see Fig. 11 (a)) and (iii) determine whether ice grows or melts when only a few hundred water molecules melt or freeze. Proceeding with such caution we estimate that the $\mathrm{NaCl}$ concentration in solution never changes more than $1 \%$ from its original value.

\section{Thermodynamic integration}

Another route to obtain the melting temperature is by finding the point at which the ice chemical potential $\left(\mu_{w}^{i}\right)$ equals the chemical potential of water in solution $\left(\mu_{w}^{s o l}\right)$. To find such point we compute separately $\mu_{w}^{i}-\mu_{w}^{0}$ and $\mu_{w}^{s o l}-\mu_{w}^{0}$, where $\mu_{w}^{0}$ is the chemical potential of pure water. The temperature at which these two chemical potential differences become equal is the melting point. To obtain chemical potential differences we use the GibbsHelmholtz equation:

$$
\left(\frac{\partial\left(\mu_{w}^{\alpha} / T\right)}{\partial T}\right)_{p}=-\frac{\bar{h}_{w}^{\alpha}}{T^{2}}
$$

Where the superscript $\alpha$ stands for a given phase (pure water, $w$, ice, $i$, or solution, sol).

$\bar{h}_{w}^{\alpha}$ is the water partial molar enthalpy which, for ice or pure water, is simply the molar enthalpy. For the solution, however, $\bar{h}_{w}^{s o l}$ is defined as:

$$
\bar{h}_{w}^{s o l}=N_{A}\left(\frac{\partial H}{\partial N_{w}}\right)_{N_{N a C l, p, T}} \approx N_{A}\left(\frac{\Delta H}{\Delta N_{w}}\right)_{N_{N a C l, p, T}}
$$

where $N_{A}$ is the Avogadro's number and $H$ is the system's enthalpy. We numerically evaluate the derivative above by computing the enthalpy for two different systems that have the same number of $\mathrm{NaCl}$ ion pairs and a different number of water molecules. It is important to make sure that the $\mathrm{NaCl}$ concentration in both systems (1.836 and $1.863 \mathrm{~m}$ in our case) narrowly encloses the concentration of interest $(1.85 \mathrm{~m})$. By dividing the enthalpy difference, $\Delta H$, by the difference of the number of water molecules, $\Delta N_{w}$, we get an estimate of $\bar{h}_{w}^{s o l}$ at $1.85 \mathrm{~m}$. Obtaining $\Delta H$ requires running long simulations (from $800 \mathrm{~ns}$ at $190 \mathrm{~K}$ to $100 \mathrm{~ns}$ at $300 \mathrm{~K}$ ) because the enthalpy difference between both systems is very small. In Fig. 2 we plot $h_{w}$ as a function of temperature for the solution (red dashed line) and the ice phases (black dashed line) and compare it with that of pure water (black solid line). As expected, the enthalpy of water in the ice phase is the lowest, whereas the enthalpy of pure water is lower than the partial molar enthalpy of water in the solution, reflecting the fact that the hydrogen bond network is disrupted by the ions.

Once the temperature dependence for the (partial) molar enthalpy is known, the water chemical potential difference between phases $\alpha$ and $\beta$ at temperature $T_{B}$ can be obtained by integrating Eq. 1 from $T_{A}$, a temperature of known chemical potential difference, to $T_{B}$ :

$\frac{\mu_{w}^{\alpha}\left(T_{B}\right)-\mu_{w}^{\beta}\left(T_{B}\right)}{T_{B}}=\frac{\mu_{w}^{\alpha}\left(T_{A}\right)-\mu_{w}^{\beta}\left(T_{A}\right)}{T_{A}}-\int_{T_{A}}^{T_{B}} \frac{\bar{h}_{w}^{\alpha}-\bar{h}_{w}^{\beta}}{T^{2}} d T$

For $\alpha=i$ and $\beta=0$ (corresponding to ice and pure water) we use $T_{A}=T_{m}^{0}$, the melting temperature of pure water $(250 \mathrm{~K}$ for TIP4P/2005), at which the chemical potential difference is $0 . \mu_{w}^{i}-\mu_{w}^{0}$ as a function of temperature is shown in Fig. 3 (black line) and has been previously reported by some of the authors [17, 40].

For $\alpha=$ sol and $\beta=0$ we use $T_{A}=298 K$, where the chemical potential difference $\mu_{w}^{\text {sol }}-\mu_{w}^{0}$ is equal to $R T \ln a_{w}(298 K)$. The activity of water at $298 \mathrm{~K}$, $a_{w}(298 K)$, has been reported in Ref. [30] as a function of the concentration for the same solution model used here. The value we take for $1.85 \mathrm{~m}$ is 0.93 . The red curve in Fig. 3] is $\mu_{w}^{s o l}-\mu_{w}^{0}$. 

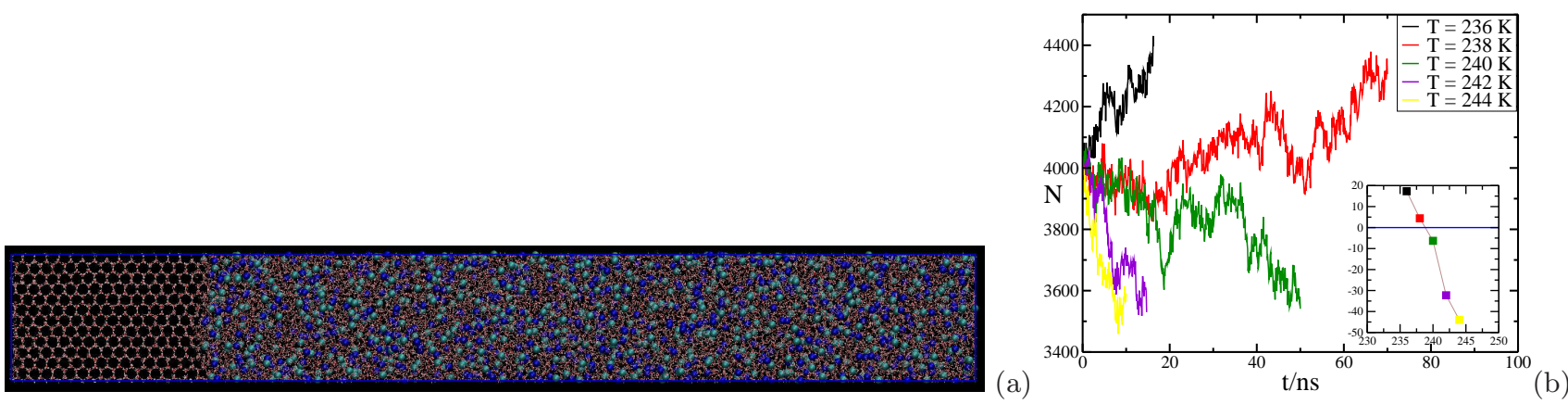

FIG. 1. (a) Snapshot of the starting configuration used to evaluate the coexistence temperature between ice Ih and the NaCl aqueous solution. The solution is elongated in order to minimize concentration changes as the ice slab grows/shrinks.(b) Main: number of molecules belonging to the ice slab as a function of time at different temperatures. Inset: slope of a linear fit to the curves in the main panel as a function of temperature. The melting temperature is taken as that for which the interpolated slope is zero $(239 \mathrm{~K})$.

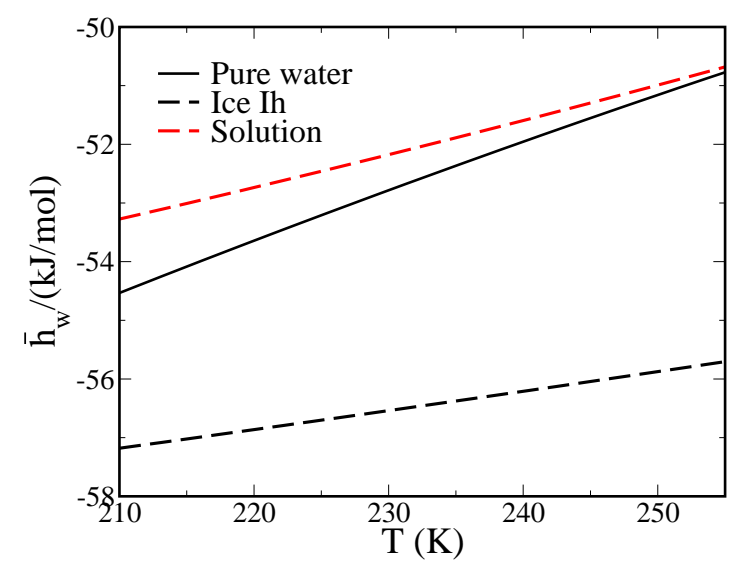

FIG. 2. Molar enthalpies of ice, pure water, and partial molar enthalpy of water in the studied $\mathrm{NaCl}$ solution as a function of temperature.

The point where both curves in Fig. 3 cross corresponds to the melting temperature, $242 \mathrm{~K}$. This value is different, but within the error bar, of that obtained by means of direct coexistence, $239 \mathrm{~K}$. The error in the direct coexistence method comes from the stochasticity associated with the finite size of the system [52]. We have used the average value between direct coexistence and the chemical potential route, $240.5 \mathrm{~K}$, as the melting temperature for all calculations in the paper.

\section{B. Critical cluster size, $N c$}

The size of the critical clusters is determined simulating ice seeds embedded in a supercooled solution. All inserted seeds have spherical shape and ice-Ih structure. Spherical ice Ih clusters (or staking mixtures of ice-Ih and ice-Ic) seems to be the nucleation pathway followed in homogeneous ice nucleation from pure water [17, 26, 67].

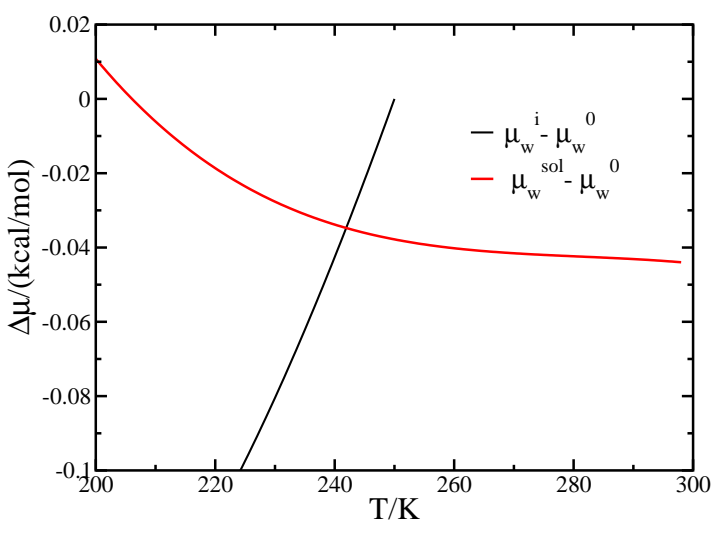

FIG. 3. Chemical potential differences (see legend) as a function of temperature. The crossing point corresponds to the melting temperature.

We insert pure ice seeds with no ions in the crystal lattice. This approximation is inspired by the fact that the brine coexists with pure ice in the experimental phase diagram [68]. To check this approximation we have performed a $260 \mathrm{~ns}$ direct coexistence simulation below the melting temperature and computed the fraction of ions coming into the newly grown ice lattice. Such fraction was smaller than 0.2 per cent. This value, which is consistent with previous simulation work 22, 24], is sufficiently small to justify the approximation of not including ions in the ice seeds.

In Ref. [66] (Fig. 7) we describe in some detail how to equilibrate an initial configuration of an embedded crystal seed into a supercooled fluid to start up the calculation. In Table I we give details on the three different initial configurations prepared ranging from a cluster of about 800 molecules up to one about 10 times larger. Starting from an equilibrated configuration with an ice cluster of $N_{c}$ water molecules we launch several isotropic $N p T$ simulations and monitor the number of ice 


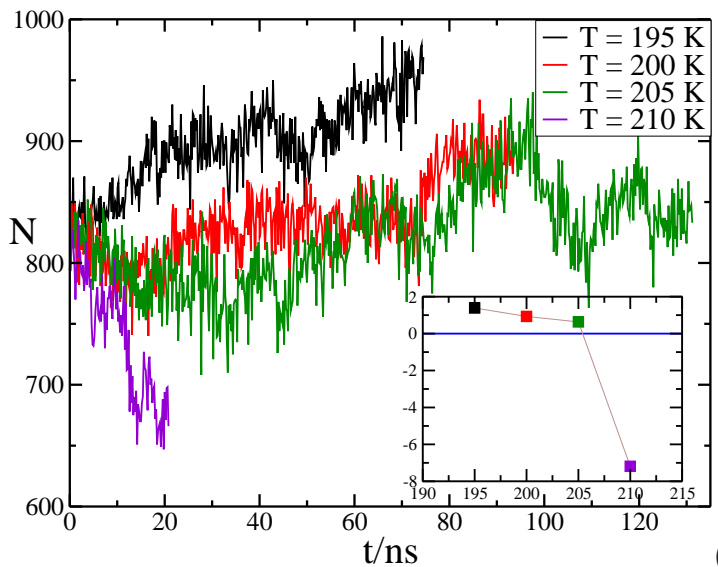

(a)
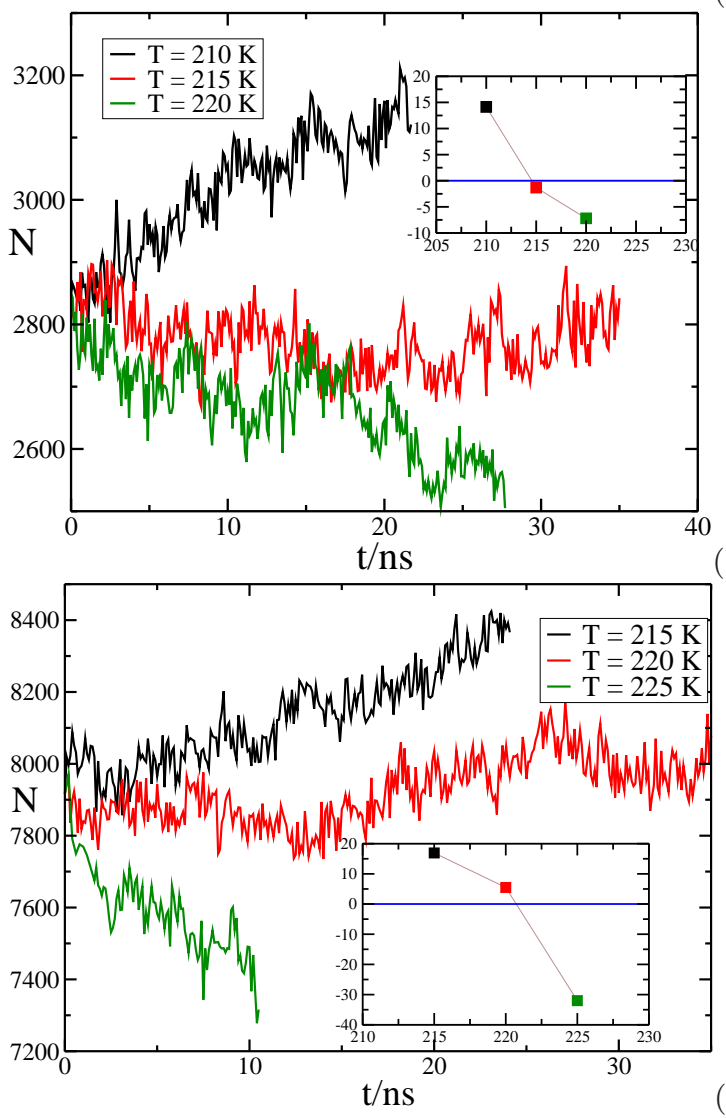

FIG. 4. Time evolution of the number of particles in the ice cluster for different inserted clusters and temperatures. Inset: Slope of a linear fit of $\mathrm{N}(\mathrm{t})$ as a function of temperature. The temperature at which the inserted cluster is critical is taken as that at which the interpolated slope is zero. The size of the inserted clusters and the temperatures at which they are found to be critical are reported in table —

molecules in the cluster, $N$. Such number is determined as explained in appendix $\mathrm{A}$. If $N$ decreases, the inserted cluster is subcritical at the chosen temperature, whereas if $N$ increases the cluster is post-critical. In such way we can determine the temperature at which the inserted cluster is critical. This is similar in spirit to the way the melting temperature is determined in direct coexistence simulations. Again, the large system sizes employed ensure that there are negligible concentration changes as the cluster grows or melts, which is the experimentally relevant case for ice nucleation in salty solutions at constant pressure and temperature.

In Figs. 4(a), (b) and (c) we show $N$ versus time for the three simulated ice clusters at different temperatures. As for the determination of $T_{m}$, from the temperature at which the slopes of linear fits to $N(t)$ interpolate to 0 we determine the temperature at which the inserted ice cluster is critical. The slope of $N(t)$ as a function of temperature is shown in the insets of Figs. 4(a), (b) and (c). The results for the temperature at which the clusters are found to be critical are reported in Table I and shown in Fig. 5(a) as a function of the supercooling, $\Delta T=T_{m}-T$. For comparison, we also include in the figure the results for pure water refined from our previous work with more statistics in the seeding simulations 17, 40]. As shown in Fig. 5(a), for a given supercooling, the number of particles needed to form a critical cluster is larger in solution than in pure water. This suggests that, apart from the trivial effect of lowering $T_{m}$, the presence of salt hinders the nucleation of ice by changing the thermodynamic or the kinetic parameters that affect ice nucleation.

To make sure that the interface between the inserted ice cluster and the solution is properly equilibrated at the beginning of the simulations shown in Fig. 4 we examine the density profile of the ions along the radial distance from the ice cluster centre of mass. We analyse the trajectory corresponding to $215 \mathrm{~K}$ in Fig. 4(b) because the cluster size stayed roughly constant at about 2800 molecules throughout the run. In Fig. [6 we compare the ions density profile in the first nanosecond of the trajectory (turquoise curve) to that corresponding to the last $10 \mathrm{~ns}$ (black curve). The ion density profile is 0 in the interior of the cluster and increases at the interface up to the equilibrium bulk density: the ice cluster is characterised by a $3 \mathrm{~nm}$ radius and an interface width of nearly $1 \mathrm{~nm}$. The ion density is surely equilibrated in the later period, starting at $25 \mathrm{~ns}$ of the trajectory, because the time required for ions to diffuse their own diameter at $215 \mathrm{~K}$ is about $2.5 \mathrm{~ns}$. It is clear from Fig. 6 that both density profiles are quite similar, both at the interface and away from the ice cluster, which proves that we have started the trajectory from a configuration where the ice cluster is surrounded by an equilibrium distribution of ions.

\section{Driving force for ice nucleation}

According to Classical Nucleation Theory, the free energy change associated to the formation of a crystal cluster with $N$ molecules is given by two competing terms:

$$
\Delta G(N)=-N\left|\Delta \mu_{w}\right|+\gamma A
$$




\begin{tabular}{cccccccccc} 
System & $N_{c}$ & $T_{c}(\mathrm{~K})$ & $\Delta T(\mathrm{~K})$ & $\Delta \mu(\mathrm{kcal} / \mathrm{mol})$ & $\rho_{w} /\left(\mathrm{g} / \mathrm{cm}^{3}\right)$ & $\gamma(\mathrm{mN} / \mathrm{m})$ & $\Delta G_{c} /\left(k_{B} T\right)$ & $f^{+} / s^{-1}$ & $\log _{10}\left(\mathrm{~J}\left(\mathrm{~m}^{-3} s^{-1}\right)\right)$ \\
\hline Sol & 794 & 205.5 & 35.0 & 0.145 & 0.980 & 28.6 & 141 & $2.69 x 10^{10}$ & -23 \\
Sol & 2850 & 214.5 & 26.0 & 0.111 & 0.982 & 33.4 & 371 & $2.10 x 10^{11}$ & -122 \\
Sol & 7916 & 220.5 & 20.0 & 0.087 & 0.983 & 36.8 & 784 & $8.40 x 10^{11}$ & -301 \\
W & 539 & 221.25 & 28.75 & 0.110 & 0.961 & 18.9 & 67 & $1.30 x 10^{11}$ & 8 \\
W & 3117 & 232.5 & 17.5 & 0.071 & 0.974 & 21.9 & 240 & $1.22 x 10^{12}$ & -66 \\
W & 7900 & 237.0 & 13.0 & 0.055 & 0.978 & 23.0 & 461 & $3.00 x 10^{12}$ & -163
\end{tabular}

TABLE I. Variables involved in the calculation of the ice Ih nucleation rate in pure and salty water. See main text for the meaning of all variables, whose values are reported with the corresponding units. The melting temperatures at 1 bar for pure water and the studied $1.85 \mathrm{~m} \mathrm{NaCl}$ solution are 250 and $240.5 \mathrm{~K}$, respectively.
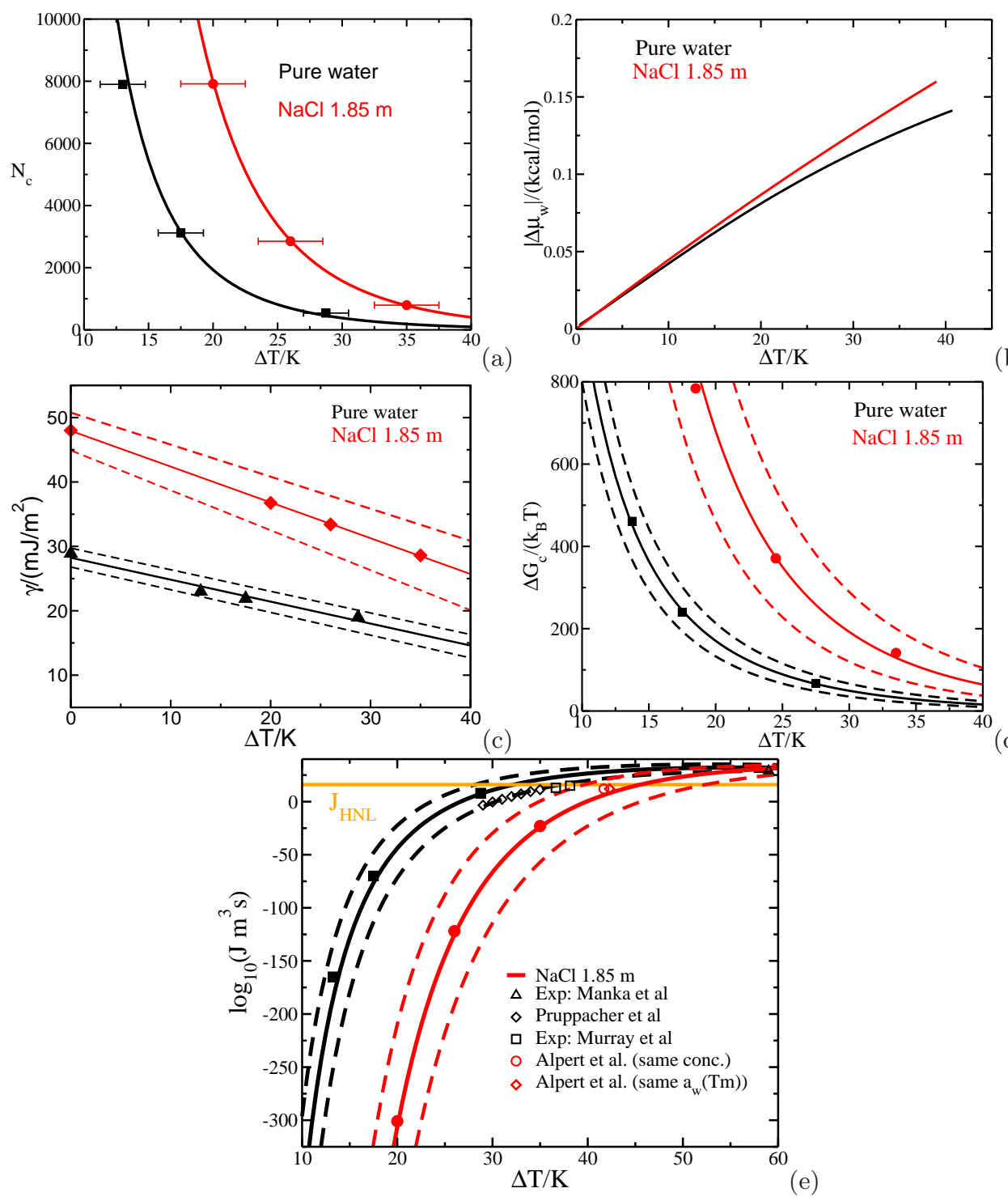

FIG. 5. Plotted for pure (black) and $1.85 \mathrm{~m}$ salty water (red) as a function of the supercooling:(a) Number of particles in the critical cluster; (b) Water chemical potential difference between the liquid and the solid phases; (c) ice-water interfacial free energy; (d) height of the ice nucleation free energy barrier; (d) decimal logarithm of the nucleation rate for pure water and the $1.85 \mathrm{~m} \mathrm{NaCl}$ solution. $J_{H N L}$ is the homogeneous nucleation line corresponding to a rate of $\mathrm{J}=10^{16} \mathrm{~m}^{-3} \mathrm{~s}^{-1}$ measured in typical experiments [69]. Solid symbols are our seeding results and empty symbols are experimental results by Pruppacher [70], Murray et al. 71], Alpert et al. [7] and Manka et al. (triangle at the top right of the figure) [72] as indicated in the legend.

The first term favours the formation of the cluster and takes into account the fact that, below $T_{m}$, the chem- ical potential of water in ice is lower than in solution. 


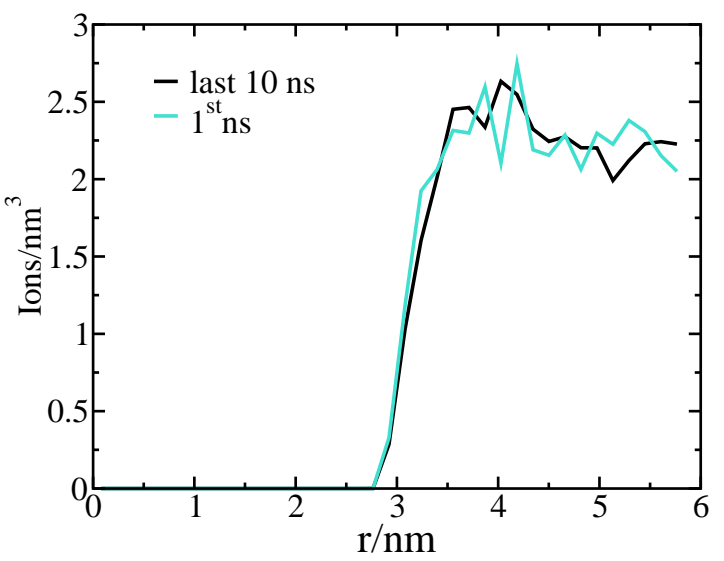

FIG. 6. Ion density as a function of the radial distance from the center of mass of the ice cluster. Turquoise, density profile averaged over the first nanosecond of the trajectory in Fig. 4 (b) corresponding to $215 \mathrm{~K}$. Black, density profile averaged over the last $10 \mathrm{~ns}$ of the same trajectory.

Therefore, the chemical potential difference of water in both phases, $\left|\Delta \mu_{w}\right|=\left|\mu_{w}^{i}-\mu_{w}^{s o l}\right|$ is the thermodynamic driving force for ice nucleation. The second term in the equation above hinders the nucleation of the crystal and is the product of the area of the cluster's surface, $A$, and the ice-solution interfacial free energy, $\gamma$.

To obtain $\left|\Delta \mu_{w}\right|$ as a function of temperature we use Eq. 3 with $\alpha=$ sol, $\beta=i$ and $T_{A}=T_{m}=240.5 \mathrm{~K}$, where $\mu_{w}^{s o l}-\mu_{w}^{i}=0$.

The chemical potential difference is plotted in Fig. 5(b), where we compare with our previous results for pure water. For a given supercooling, the thermodynamic driving force for the formation of ice is larger in solution (red) than in pure water (black), specially at high supercooling. This is consistent with the fact that the partial enthalpy of water in solution is higher than the enthalpy in pure water (Fig. 22). Therefore, the free energy gain when a fluid water molecule becomes part of the ice cluster is larger in solution than in pure water. According to $\left|\Delta \mu_{w}\right|$, then, the formation of ice in solution should be easier than in pure water, which is not consistent with Fig. 5(a), where we show that, for a given supercooling, larger clusters are required to nucleate ice in solution than in pure water. However, $\left|\Delta \mu_{w}\right|$ is not the full story. One also has to take into account the ice-solution interfacial free energy, $\gamma$, which is what we discuss in the following section.

\section{Ice-solution interfacial free energy}

By maximizing Eq. 4 and assuming a spherical cluster shape the following expression can be found for the

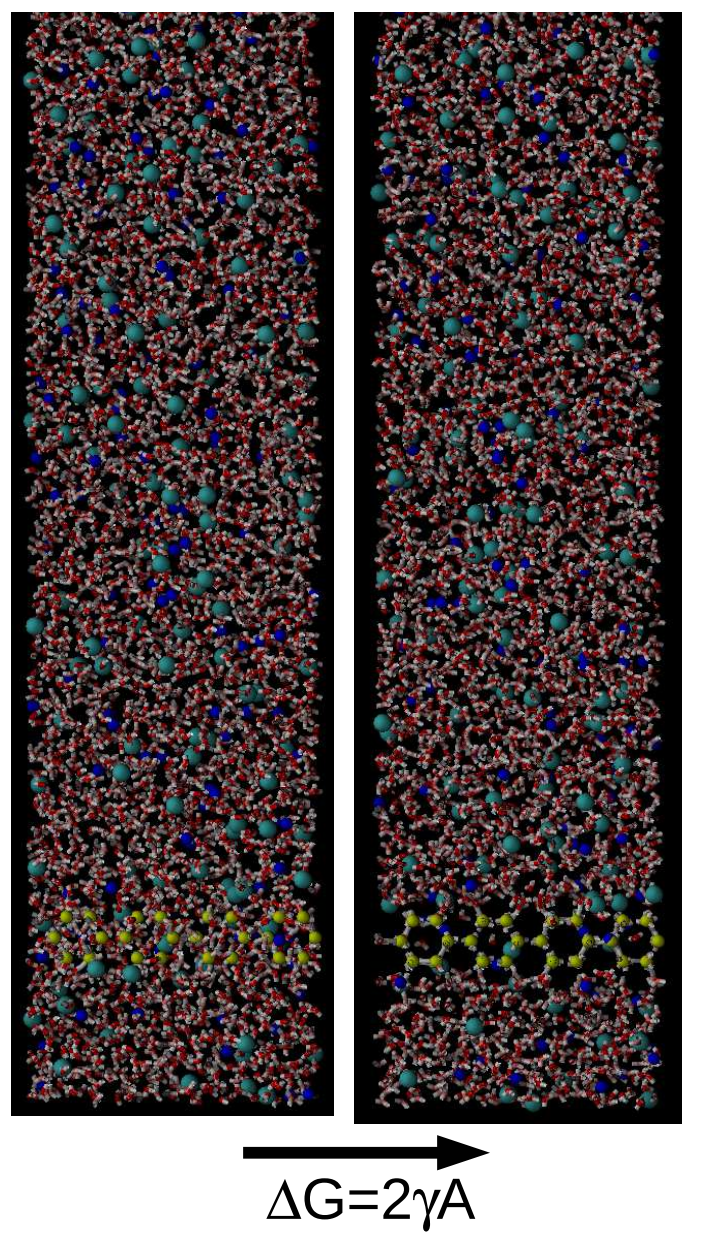

FIG. 7. Snapshot of a $1.85 \mathrm{~m} \mathrm{NaCl}$ solution configuration at the melting temperature at 1 bar. The square well-like interaction between the mold sites (yellow particles) and the oxygens of the water molecules is switched off in the left panel and on in the right panel. Note that the number for crystal planes in the mold, $N_{l}$, is three in this case.

interfacial free energy:

$$
\gamma=\left(\frac{3 N_{c} \rho_{s}^{2}\left|\Delta \mu_{w}\right|^{3}}{32 \pi}\right)^{1 / 3},
$$

which depends on the number of particles in the critical cluster, $N_{c}$, the ice number density, $\rho_{s}$, and the icesolution water chemical potential difference, $\left|\Delta \mu_{w}\right|$. In Table 【 we give the calculated values of $\gamma$ for the three studied cluster sizes. Both in the table and in Fig. 5(c) we show that the ice-solution interfacial free energy is significantly larger than the ice-water one (for a given $\Delta T)$. Therefore, despite the fact that the bulk free energy drop associated to the formation of an ice cluster is larger in solution than in pure water (Fig. 5(b)), the increase of surface free energy is higher for the solution (Fig. [5(c)), which explains why salt hinders the nucleation of ice clusters (Fig. 5(a)). In fact, once both $\gamma$ and 
$\Delta \mu$ are known, the height of the nucleation free energy barrier can be evaluated according to CNT as:

$$
\frac{\Delta G_{c}(T)}{k_{B} T}=\frac{16 \pi(\gamma(T))^{3}}{3\left(\rho_{s}(T)\right)^{2}\left|\Delta \mu_{w}(T)\right|^{2} k_{B} T}
$$

The calculated values are reported in Table 1 and plotted in Fig. 5(d). Clearly, for a given supercooling more work is required to reversibly grow an ice cluster in solution than in pure water. The responsible for such increase is the interfacial free energy, given that $\Delta \mu_{w}$ actually contributes to lower $\Delta G_{c}$.

To make sure that $\gamma$ increases when adding salt we also compute $\gamma$ for a flat interface (at coexistence) using the Mold Integration method [38] that we have recently employed to compute the ice-water interfacial free energy for pure water at ambient [39] and high pressure [26]. In this method, which is only valid for coexistence conditions, a mold composed of square wells placed in the lattice sites of one or several crystal planes is gradually switched on to induce the formation of a solid slab in the fluid at coexistence conditions. The idea is sketched in Fig. 17. We note that the interfacial crystal halo generated by the mold is free to fluctuate and to incorporate and expel ions to reach its equilibrium structure. The free energy difference between the fluid and the fluid with the structure induced by the mold can be computed by integrating the average number of filled wells, $\left\langle N_{f w}\right\rangle$, along such thermodynamic path:

$$
\Delta G=N_{w} \epsilon_{m}-\int_{0}^{\epsilon_{m}}\left\langle N_{f w}>d \epsilon\right.
$$

where $N_{w}$ is the number of wells in the mold, $\epsilon$ is the well depth and $\epsilon_{m}$ is the maximum well depth $\left(10 k_{B} T\right.$ in our case). When the well radious, $r_{w}$, is equal to a certain "optimal" value, $r_{w}^{o}, \Delta G$ is equal to $2 \gamma A$ [38], where $A$ is the area of the mold (the factor of 2 comes from the fact that two interfaces are generated). The position of the wells is fixed during the simulation and only the side of the simulation box perpendicular to the interface fluctuates to keep the pressure constant. Mold Integration can be easily implemented in GROMACS using a tabulated interaction potential between the mold and the fluid particles [38]. A cut-off of $14 \AA$ is used for all interactions. The interfacial free energy of different crystal orientations can be obtained by using molds corresponding to different crystal planes [38, 39]. In this work we study the basal, primary prismatic (pI) and secondary prismatic (pII) orientations. In table I we report details on our Mold Integration calculations such as $N_{w}, A, r_{w}^{o}$ or the number of crystal planes in the mold, $N_{l}$, for each crystal orientation studied.

In order to find $r_{w}^{o}$ we run several simulations for different well radii monitoring the number of ice-like molecules. The simulations start from a configuration of the solution at coexistence conditions ( 1 bar and $240.5 \mathrm{~K}$ ) and the mold is switched on at the beginning of the simulation. $r_{w}^{o}$ is enclosed between the largest radious for which ice

\begin{tabular}{ccccccc}
\hline System & Plane & $\left(L_{y} L_{z}\right) / \AA^{2}$ & $N_{w}$ & $N_{l}$ & $r_{w}^{0} / \AA$ & $\gamma /\left(m J / m^{2}\right)$ \\
\hline \hline Sol & basal & 1127.7 & 192 & 3 & $0.97(10)$ & $43(2)$ \\
Sol & pI & 1063.2 & 192 & 3 & $0.72(10)$ & $49(3)$ \\
Sol & pII & 920.73 & 192 & 3 & $0.85(10)$ & $52(3)$ \\
Sol & $\gamma_{0}$ & & & & & $48(3)$ \\
\hline W & basal & 1128.7 & 128 & 2 & $0.83(5)$ & $27.2(8)$ \\
W & pI & 1064.1 & 128 & 2 & $0.73(5)$ & $29.5(8)$ \\
W & pII & 921.55 & 128 & 2 & $0.94(5)$ & $30.0(8)$ \\
W & $\gamma_{0}$ & & & & & $28.9(8)$ \\
\hline \hline
\end{tabular}

TABLE II. Computational details of the Mold Integration calculations performed to obtain the ice-solution interfacial free energy for different crystal orientations. For comparison, we also report the results for pure water (W) from Ref. [39]. $\gamma_{0}$ is the average of the three orientations. $N_{l}$ is the number of crystal layers in the mold.

grows with no induction period and the smallest one for which ice either does not grow, or grows after some induction time [38]. Such runs for the pII plane are shown in Fig. 8)(a). For $r_{w}=0.7 \AA$ (blue curves) or smaller (not shown) ice grows with no induction period in all trajectories, whereas for $r_{w}=1 \AA$ (green curves) or larger (not shown) ice does not grow. Thus, for this case we set $r_{w}^{o}=0.85 \pm 0.10 \AA$.

Once we set a value for $r_{w}^{o}$ we obtain $\Delta G$ from Eq. 7 for several values of $r_{w}$ larger than $r_{w}^{o}$. The integrand of Eq. 7 for the pII orientation is shown in Fig. 8(b) for several $r_{w}$ values. Each point in Fig. 8(b) is obtained in a simulation with well depth $\epsilon$ (indicated by the $\mathrm{x}$-axis in the figure). By integrating these curves we obtain $\gamma\left(r_{w}\right)=\Delta G\left(r_{w}\right) /(2 A)$, as shown in Fig. 8(c) for all studied orientations. Filled symbols correspond to our calculations for $r_{w}>r_{w}^{o}$ and empty ones to a linear extrapolation to $r_{w}=r_{w}^{o}$, that gives the value for $\gamma$. As in the case of pure water, the interfacial free energy of the prismatic planes is higher than that of the basal plane. In fact, the $\gamma$ anisotropy is higher for salty than for pure water: in the case of pure water the interfacial free energy of the prismatic planes is about 2-3 $\mathrm{mN} / \mathrm{m}$ higher than that of the basal plane, whereas for salty water the difference is about $6-9 \mathrm{mN} / \mathrm{m}$. For all studied planes, the solid-fluid interfacial free energy is higher in the solution than in pure water. Therefore, the Mold Integration method confirms the seeding prediction that the ice-fluid interfacial free energy is larger for the solution. The orientationally averaged $\gamma$ from the Mold Integration calculations is plotted alongside the seeding results in Fig. 5(c). Within our statistical uncertainty, the value from Mold Integration $(\Delta T=0)$ is consistent with the seeding calculations $(\Delta T>0)$. 

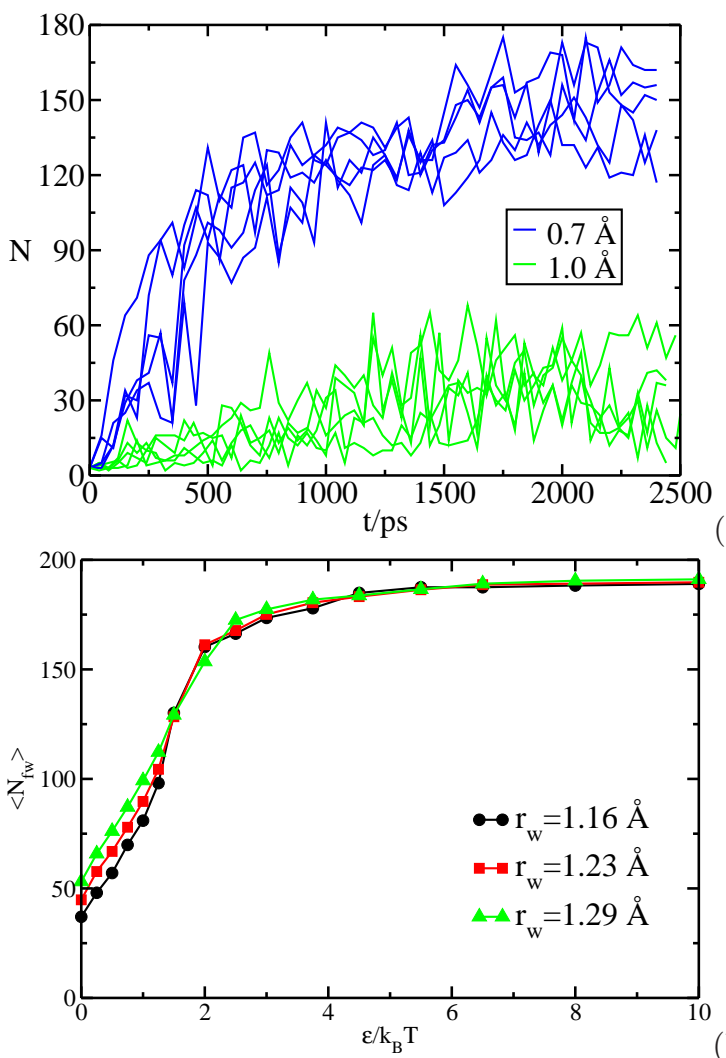

(a)

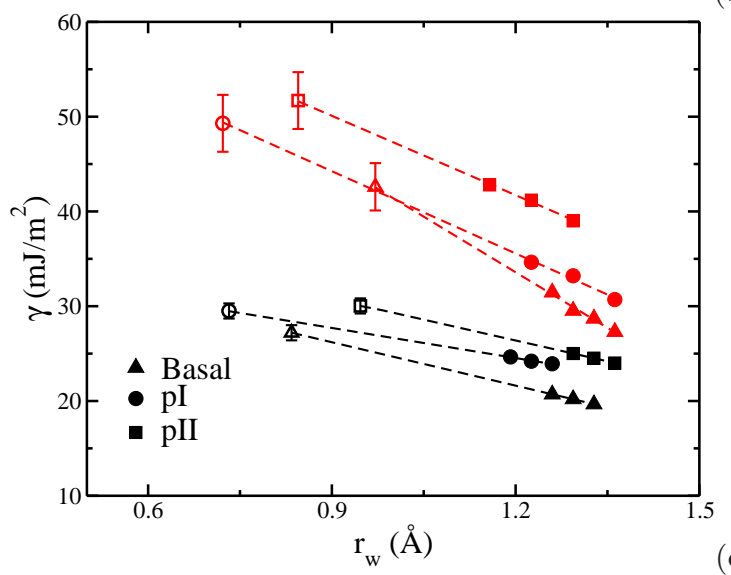

(b)

FIG. 8. (a) Number of ice-like molecules growing from the mold as a function of time for several trajectories starting from the studied $\mathrm{NaCl}$ solution at coexistence conditions. The mold is switched on at the beginning of the simulation. The well radious $r_{w}$ is $1 \AA$ for the green curves and $0.7 \AA$ for the blue ones. (b) Integrand of Eq. 7 for several $r_{w}$ values, as indicated in the legend, for the secondary prismatic plane. (c) Ice-fluid interfacial free energy as a function of $r_{w}$ for several crystal orientations as indicated in the legend. Red data correspond to the solution and black ones, from Ref. [39], to pure water. Filled symbols are the results of our calculations and empty ones are the extrapolation to the corresponding $r_{w}^{o}$, which give the final $\gamma$ values.
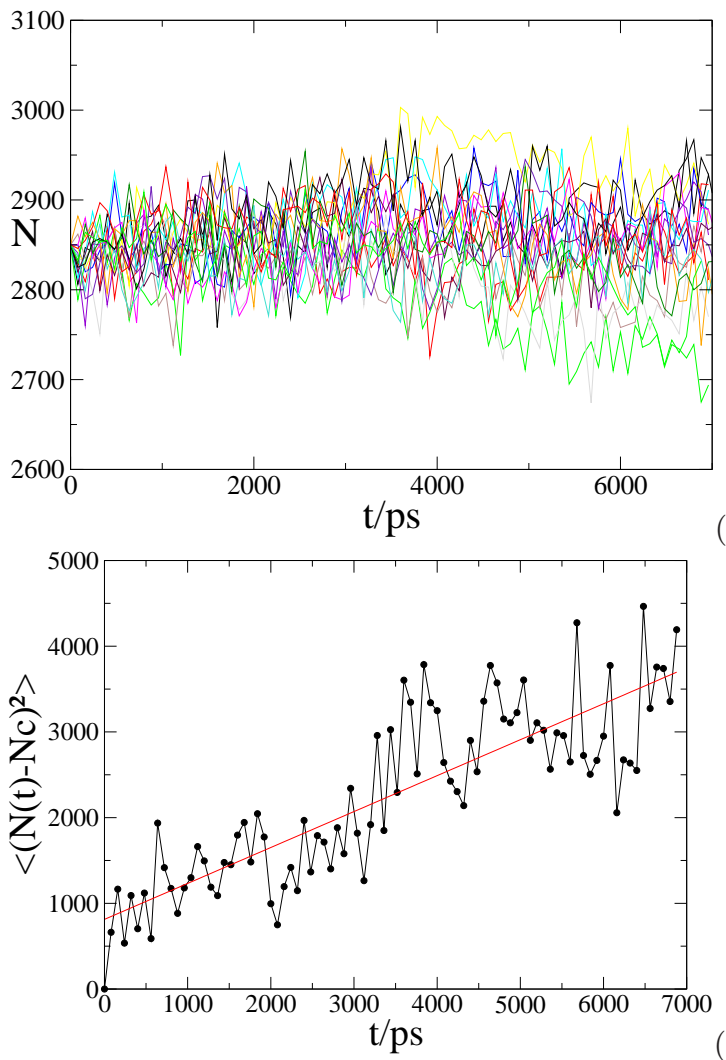

(a)

FIG. 9. (a) Time evolution of the number of particles, $N$, in a critical ice cluster of $\sim 2850$ molecules in salty water. Different trajectories correspond to runs launched with different velocity distributions. (b) Mean squared difference of the number of molecules in the cluster at time $t$ and time 0 as a function of time.

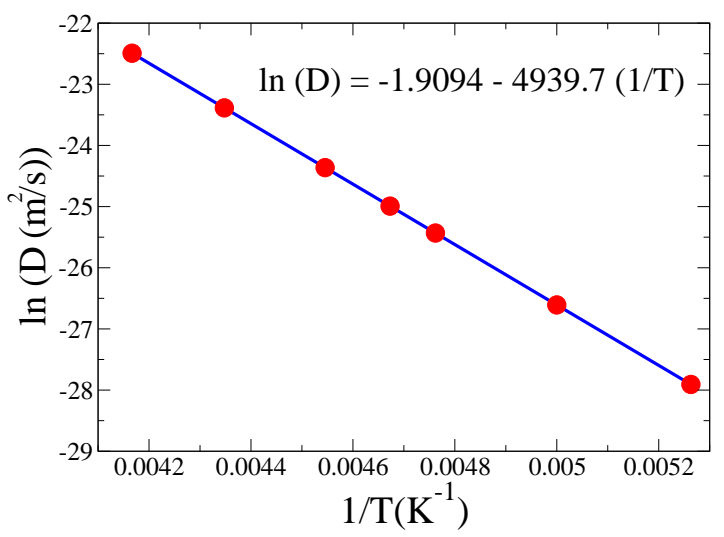

FIG. 10. Diffussion coefficient of water in the salty solution as a function of the inverse temperature.

\section{E. Nucleation rate}

We have already shown in Fig. 5(a) that, for a given $\Delta T$, the size of critical ice clusters is larger in salty than 
in pure water. In the previous section we argue that this is due to the increase of the ice-liquid interfacial free energy. In the present section we aim at quantifying the extent to which ice nucleation is slowed down by adding salt. The speed of ice nucleation is measured by the nucleation rate, $J$, that is simply defined as the number of critical ice clusters appearing per unit time and volume. According to CNT $J$ is given by:

$$
J=A e^{-\Delta G_{c} /\left(k_{B} T\right)},
$$

where $\Delta G_{c}$ has already been computed from Eq. 6] (see Fig. [5(d)). A, the kinetic pre-factor, is given by:

$$
A=\rho_{w} \sqrt{\frac{\left|\Delta \mu_{w}\right|}{6 \pi k_{B} T N_{c}}} f^{+}
$$

where $\rho_{w}$ is the density of water in the solution and $f^{+}$ is the attachment rate, or the frequency with which new water molecules attach to the critical cluster, that can be calculated as 37]

$$
f^{+}=<\left(N(t)-N_{c}\right)^{2} /(2 t)>
$$

Computing $f^{+}$requires performing several simulations of the critical cluster where $N$ is monitored as a function of time, as shown in Fig. 9(a). By averaging $\left(N(t)-N_{c}\right)^{2}$ over all these trajectories we obtain a curve such as that shown in Fig. 9(b), whose slope is $2 f^{+}$.

The calculation of $f^{+}$is rather involving and we have only performed it as described above for the cluster containing $N_{c}=2850$ molecules. For the other two studied clusters we have used the following expression for $f^{+}$provided by CNT:

$$
f^{+}=\frac{24 D N_{c}^{2 / 3}}{\lambda^{2}}
$$

where $D$ is the diffusion coefficient of water in solution, which we plot as a function of temperature in Fig. 10. and $\lambda$ is the distance travelled by particles in the vicinity of the cluster's surface to attach to the cluster. By equating the value of $f^{+}$obtained via Eq. 10 for the cluster with 2850 molecules to Eq. 11 we obtain $\lambda=6 \AA$, which is a reasonable value of the order of the molecular diameter. We use this value of $\lambda$ combined with Eq. 11 to estimate $f^{+}$for the other two studied clusters. The values of $f^{+}$thus obtained are reported in Table \.

We now have all factors required to compute the nucleation rate via Eq. 8. The results for the three studied clusters are reported in table \and plotted in Fig. 5(e) in comparison to those of pure water from Refs. [17, 40]. For a given supercooling, the nucleation rate is lower in salty than in pure water, as expected from the result that the critical cluster size is larger in solution (Fig. [5(a)).

\section{F. Decrease of $J$ when adding salt}

We now try to better rationalise which are the factors that contribute to the decrease of the nucleation rate
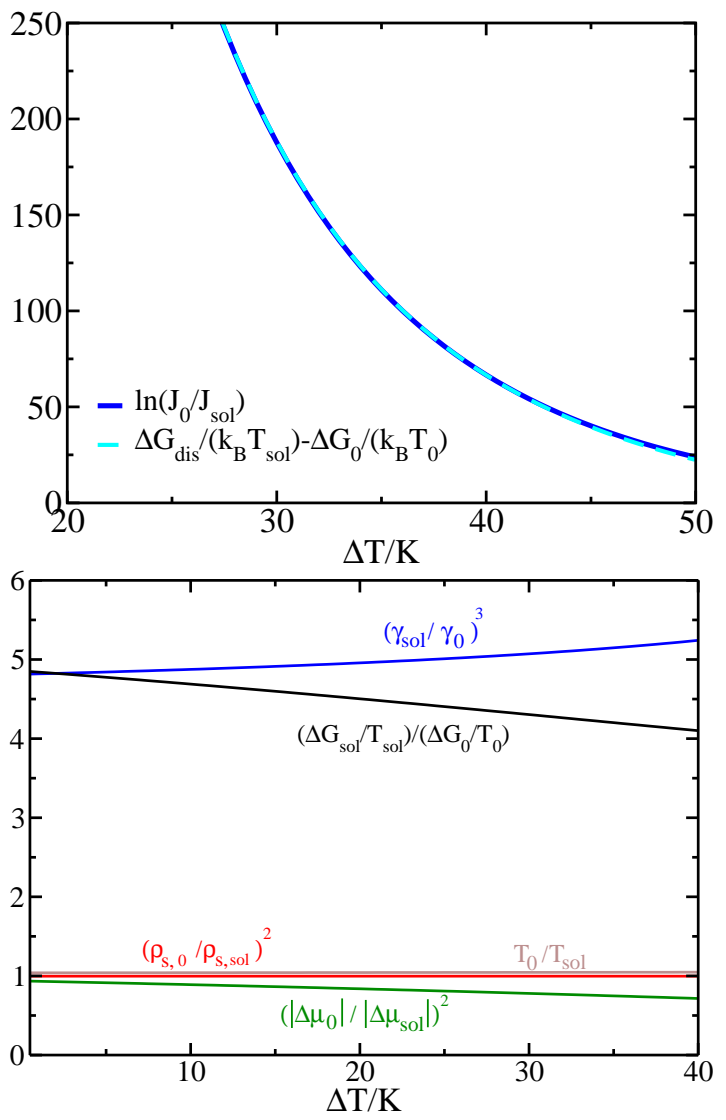

FIG. 11. (a) Difference in $\ln J$ and $-\Delta G_{c} /\left(k_{B} T\right)$ between salty and pure water as a function of the supercooling. (b) Black curve, factor by which $\Delta G_{c} /\left(k_{B} T\right)$ increases in the salty solution with respect to pure water as a function of the supercooling. Coloured curves, different factors that contribute to such increase, as indicated in the figure. The product of the coloured curves gives the black curve.

when adding salt. To do that we first need to fit the data coming from the seeding simulations, which we do using the CNT expressions above [36, 40]. In our fitting procedure we assume a linear temperature dependence of $\gamma$. We obtain the linear fit by combining seeding and Mold Integration data (see Fig. 5(c)). With $\gamma(T)$ and Eq. 5 we obtain $N(T)$ (solid lines in Fig. 5(a)). From $N(T), \Delta \mu_{w}(T)$ and Eq. 6 we obtain CNT fits to $\Delta G_{c}(T)$ (solid lines in Fig. 5(d)). Finally, with the Arrhenius-like fit to $D(T)$, shown in Fig. 10, and Eqs. 11 and 8 we get the fits to $J$ shown in Fig. 5 (e).

Having the temperature dependence of the factors that affect the nucleation rate we can quantify and compare their variation when adding salt. We perform the comparison at constant supercooling rather than at absolute temperature to get rid of the trivial lowering in $J$ caused by the decrease of the melting temperature. We compare first the extent to which the kinetic prefactor and $\Delta G_{c} /\left(k_{B} T\right)$ affect the drop in $J$. According to Eq. 8 the difference in $\ln (J)$ between pure (0) and salty (sol) water, 
$\ln \left(J_{0} / J_{s o l}\right)$, has two terms, one for the kinetic pre-factor, $\ln \left(A_{0} / A_{\text {sol }}\right)$, and one for the free energy barrier height, $\Delta G_{c}^{\text {sol }}\left(T_{\text {sol }}\right) /\left(k_{B} T_{\text {sol }}\right)-\Delta G_{c}^{0}\left(T_{0}\right) /\left(k_{B} T_{0}\right)$ (note that $T_{0}$ and $T_{\text {sol }}$ are not the same because we compare at constant $\Delta T)$. The fact that $\ln \left(J_{0} / J_{\text {sol }}\right)$ is equal to the difference in $\Delta G_{c} /\left(k_{B} T\right)$ (see Fig. 11(a)) means that the kinetic prefactor is not significantly affected by salt. It is then ratio between the free energy barrier height and the thermal energy that changes when adding salt. In Fig. 11(b), black curve, we show that $\Delta G_{c} /\left(k_{B} T\right)$ is between 4 and 5 times larger in the salty solution. According to Eq. 6 the factors that affect $\Delta G_{c} /\left(k_{B} T\right)$ are $\Delta \mu_{w}, T, \rho_{s}$ and $\gamma$. In Fig. 111(b) we show the factor by which each of them contribute to the change of $\Delta G_{c} /\left(k_{B} T\right)$ when adding salt. The ice density and the temperature have a negligible effect on the change of the free energy barrier height (red and brown curves in Fig. 11(b)). The chemical potential difference does not change the barrier at low supercooling and lowers it about thirty per cent at high supercooling (green curve in Fig. 11(b)). As previously discussed, such lowering is a consequence of the fact that the partial molar enthalpy of water in solution is higher than in pure water. Therefore, $\Delta \mu_{w}$ aids ice nucleation when salt is added. However, the interfacial free energy largely compensates the modest effect of $\Delta \mu_{w}$, given that the increase of $\gamma$ with salt multiplies by a factor of 5 the free energy barrier (blue curve in Fig. 11(b)). The effect of the increase of $\gamma$ on $\Delta G_{c} /\left(k_{B} T\right)$ is magnified by the fact that $\gamma$ goes as a third power in Eq. 6] In summary, the driving force for ice nucleation, $\Delta \mu_{w}$, increases when adding salt, however $\gamma$, that hinders ice nucleation, increases to a greater extent, which causes the salt-induced deceleration of ice nucleation.

\section{DISCUSSION}

\section{A. Comparison with the experiment}

\section{Melting point depression}

Experimentally, a $1.85 \mathrm{~m} \mathrm{NaCl}$ solution freezes $6.6 \mathrm{~K}$ below the melting point of pure water [73]. The model captures that salt decreases the ice melting temperature, predicting a $9.5 \mathrm{~K}$ depression for a $1.85 \mathrm{~m}$ solution. Such depression is caused in experiments by a $2.6 \mathrm{~m} \mathrm{NaCl}$ concentration [73]. Hence, the effect of salt on the melting point is larger in the model than in the experiment. This suggests that the $\mathrm{NaCl}$ model we are using causes a larger decrease in the chemical potential of TIP4P/2005 water than that caused by real $\mathrm{NaCl}$ in real water (see Fig. $4 \mathrm{~b}$ of Ref. [30]).

To account for such enhanced effect of the model, it is interesting to compare model and experiment for the same activity of water in the solution coexisting with ice, $a_{w}^{i}$. We can compute $a_{w}^{i}$ as:

$$
a_{w}^{i}=\exp \left(\Delta \mu_{0} /(R T)\right)
$$

where $\Delta \mu_{0}$ is the water chemical potential difference in ice and in pure liquid water at the ice-solution coexistence temperature $\left(T_{m}=240.5 \mathrm{~K}\right)$. We know $\Delta \mu_{0}$ from our previous work on homogeneous ice nucleation [17, 40]. We obtain $a_{w}^{i}=0.92$. For such $a_{w}^{i}$, a melting point of $\sim 263 \mathrm{~K}$ can be interpolated from the experimental data reported in Ref. [7], which is about $10 \mathrm{~K}$ below the melting temperature of pure water. Such melting point depression of $10 \mathrm{~K}$ compares better with the $9.5 \mathrm{~K}$ obtained with our model. Therefore, when experiment and model are compared for a given water activity, a better agreement is obtained than when they are compared at a given concentration. In order to improve the employed model for $\mathrm{NaCl}$ aqueous solutions it is therefore necessary to modify the ion-water interactions in such way that a smaller water activity drop is caused by the ions.

\section{Nucleation rate}

It is experimentally known that the supercooling required to freeze microscopic salty water drops is larger than that required to freeze pure water drops [5, 77, 69]. Equivalently, for a given supercooling, the nucleation rate is higher in pure than in salty water. In Fig. 5(e) we show that the model indeed captures the experimental trend. According to our results, such trend is due to an increase of the ice-solution interfacial free energy when adding salt. This simulation prediction is quite valuable considering the lack of accuracy in experimental measurements/estimates of the ice-liquid interfacial free energy [8].

According to the employed model (Fig. 5(e)), the nucleation rates in pure and salty water are quite similar at very high supercooling (about $60 \mathrm{~K}$ ). The rate in such conditions can be measured using nanoscopic drops 72 . Consistently with the similarity of the rate, the interfacial free energy of pure water is also predicted to be similar to that of salty water at high supercooling (see Fig. [5](c)).

The model predictions for the nucleation rate in salty water, shown in Fig. 5(e), can be directly compared to experimental measurements by Alpert et al. 7] (empty symbols). The comparison can be made either for a solution with the same $\mathrm{NaCl}$ concentration (empty circle) or for one with the same water activity at the melting temperature, $a_{w}\left(T_{m}\right)$ (empty diamond). In either case the agreement between simulation and experiment is quite satisfactory.

\section{SUMMARY AND CONCLUSIONS}

We use computer simulations to investigate the effect of salt on homogeneous ice nucleation. To study the aqueous solution, we use the TIP4P/2005 model for water in combination with the Joung Cheetham $\mathrm{NaCl}$ model as parametrised for $\mathrm{SPC} / \mathrm{E}$ water. To start with, we 
compute the ice melting point for the model using both direct coexistence and thermodynamic integration from previous calculations of the chemical potential of water in solution. The model predicts a larger cryoscopic depression than the experimental one for a given salt concentration. However, if we compare experiment and simulation for the same activity of water at coexistence we obtain a good agreement for the melting point drop. This suggests that the employed $\mathrm{NaCl}$ model is affecting the solute (water) to a greater extent than real $\mathrm{NaCl}$ does.

After computing the melting temperature we compute the size of critical ice clusters by embedding spherical ice Ih seeds in the supercooled solution and simulating trajectories at different temperatures. We compute the chemical potential difference of water in ice and in the solution using thermodynamic integration of the partial molar enthalpy from the melting temperature. With such chemical potential difference and the number of particles in the critical cluster we obtain the height of the ice nucleation free energy barrier and the ice-solution interfacial free energy using the expressions provided by Classical Nucleation Theory. We also compute the ice-solution interfacial free energy at coexistence using the Mold Integration method, that provides a thermodynamic route to reversibly grow an ice slab in the fluid at coexistence conditions. The interfacial free energies obtained with seeding in supercooled conditions extrapolate linearly to the value at coexistence obtained with Mold Integration. This consistency test supports the validity of our approach. The interfacial free energy at any supercooling increases with respect to that of pure water (obtained in previous studies).

The kinetic pre-factor for the ice nucleation rate is obtained for one of the ice seeds by launching many trajectories and computing the mean squared displacement of the number of molecules in the cluster. The Classical Nucleation Theory expression for the kinetic pre-factor, which is proportional to the diffusion coefficient of water molecules in the liquid, is consistent with such calculation. Such consistency enabled us to use the diffusion coefficient of water as a function of temperature to get the temperature dependence of the kinetic pre-factor. That, combined with the temperature dependence obtained for the ice-solution chemical potential difference of water and for the interfacial free energy, gave us the temperature dependence of the nucleation rate for a wide range of orders of magnitude.

The model qualitatively captures the experimental trend that, for a given supercooling, the nucleation rate decreases by adding salt. Our model predicts that salt hinders ice nucleation despite the fact that the chemical potential difference between water in ice and in solution is larger when salt is added. This would in principle favour ice nucleation for a certain supercooling. However, the increase of the interfacial free energy largely compensates for the increase of the thermodynamic driving force for nucleation and the net effect is a deceleration of the ice nucleation process. The ice nucleation rate predicted by the salty water model is in good agreement with experimental measurements, which brings confidence in the predictions made by the model.

\section{Acknowledgements}

This work was funded by grants FIS2013/43209-P and FIS2016-78117-P FIS2016-78847-P of the MEC, and the UCM/Santander 910570 and PR26/16-10B-2. C.Valeriani and E. Sanz acknowledge financial support from a Ramon y Cajal Fellowship. J. R. Espinosa acknowledges financial support from the FPI grant BES2014-067625. Calculations were carried out in the supercomputer facilities La Palma and Magerit from the Spanish Supercomputing Network (RES) (projects QCM2015-3-0036 and QCM-2016-1-0039). The authors acknowledge the computer resources and technical assistance provided by the Centro de Supercomputacion y Visualizacion de Madrid (CeSViMa). 


\section{Appendix A: Determining $N$}

To determine the number of particles in the ice phase (be it the ice slab in the direct coexistence simulations or the cluster in the seeding ones) we use the $\bar{q}_{i}$ rotationally invariant local-bond order parameter proposed in Ref. [74]. $\bar{q}_{i}$ is a scalar number whose value for a given particle depends on the relative positions of the tagged particle and its neighbors within a certain distance (3.5 $\AA$, around the first minimum in the oxygen-oxygen radial distribution function of the liquid phase). We only use the oxygens for the calculation of $\bar{q}_{i}$. In Fig. 12 (a) we plot typical $\left(\bar{q}_{4}, \bar{q}_{6}\right)$ values for ice (red) and solution (green) water molecules. Differently from $\bar{q}_{4}$, it is possible to establish a threshold for $\bar{q}_{6}, \bar{q}_{6, t}$, to discriminate between ice-like and solution-like water molecules (indicated with a horizontal dashed line in Fig. 12(a)). To establish the $\bar{q}_{6, t}$ value we look at the point at which the fraction of wrongly labelled particles in both phases coincide (see Fig. 12(b)). As shown in Fig. 12(c), the value of such threshold depends on temperature. For a given temperature, particles with $\bar{q}_{6}>\bar{q}_{6, t}$ are labelled as solid-like. Having established all solid-like particles in the system, we cluster them using a neighbour cut-off distance of $3.5 \AA$. $N$ is the number of molecules in the largest detected cluster of solid-like molecules.
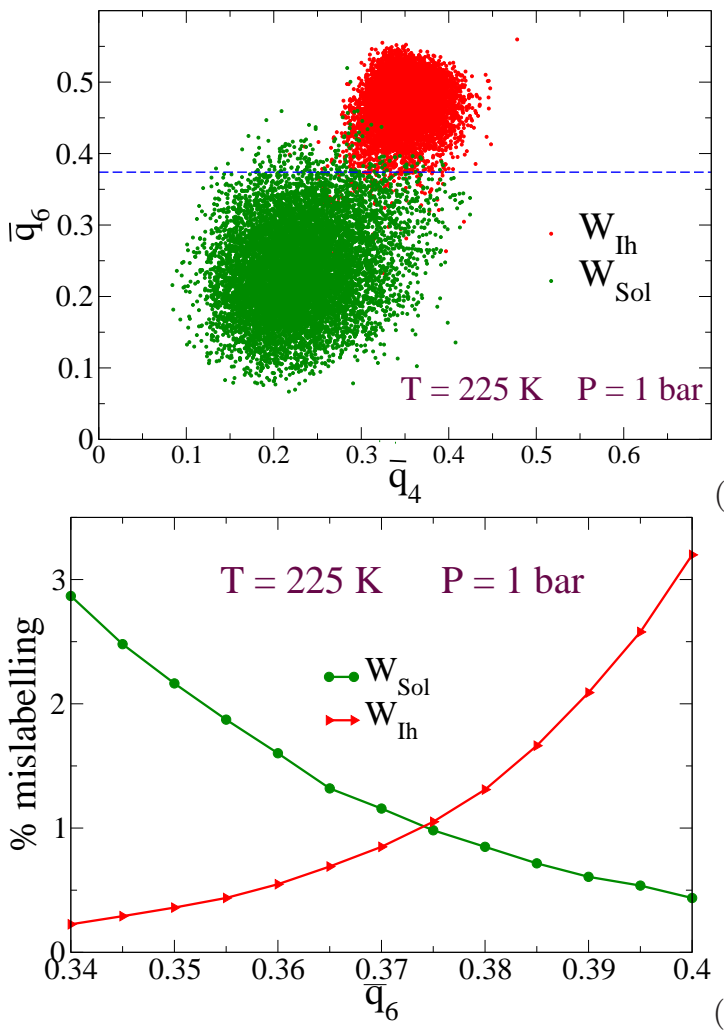

(a)

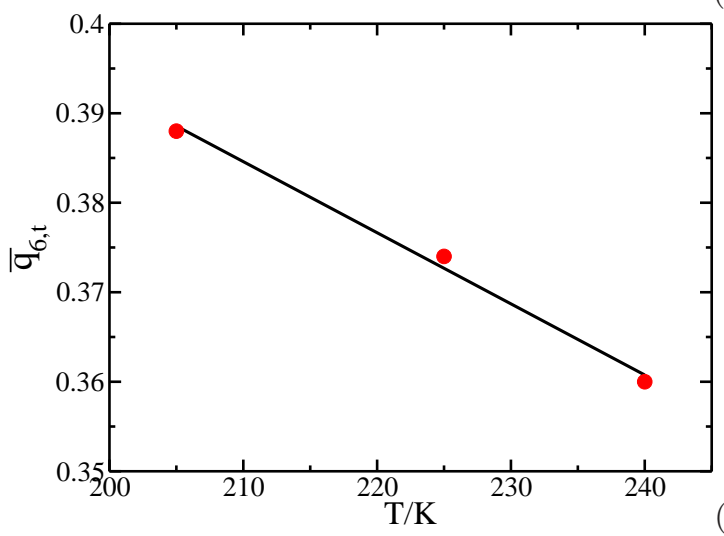

(b)

FIG. 12. (a) $\bar{q}_{6}$ vs $\bar{q}_{4}$ for water in the salty solution (green) and water in ice Ih (red). (b) Fraction of mislabelled particles as a function of the $\bar{q}_{6}$ threshold for both phases at $225 \mathrm{~K}$ and 1 bar. The $\bar{q}_{6}$ threshold is chosen at the crossing point between both curves. (c) Selected $\bar{q}_{6}$ threshold, $\bar{q}_{6, t}$, as a function of temperature. 
[1] A. G. Gerrard, Rocks and Landforms. Springer Netherlands, 1988.

[2] J. K. Li and T. C. Lee, "Bacterial ice nucleation and its potential application in the food-industry," Trends in Food Sci. Es Tech., vol. 6, pp. 259-265, 1995.

[3] L. R. Maki, E. L. Galyan, C. M.M., and D. R. Caldwell, "Ice nucleation induced by pseudomonas-syringae," Appl. Microbiol., vol. 28, pp. 456-459, 1974.

[4] G. J. Morris and E. Acton, "Controlled ice nucleation in cryopreservation-a review," Cryobiology, vol. 66, p. 85, 2013.

[5] H. Kanno and C. A. Angell, "Homogeneous nucleation and glass formation in aqueous alkali halide solutions at high pressures," The Journal of Physical Chemistry, vol. 81, no. 26, pp. 2639-2643, 1977.

[6] K. F. Kelton, Crystal Nucleation in Liquids and Glasses. Boston: Academic, 1991.

[7] P. A. Alpert, J. Y. Aller, and D. A. Knopf, "Ice nucleation from aqueous nacl droplets with and without marine diatoms," Atmos. Chem. Phys., vol. 11, p. 5539, 2011.

[8] L. Ickes, A. Welti, C. Hoose, and U. Lohmann, "Classical nucleation theory of homogeneous freezing of water: thermodynamic and kinetic parameters," Phys. Chem. Chem. Phys., vol. 17, pp. 5514-5537, 2015.

[9] G. C. Sosso, J. Chen, S. J. Cox, M. Fitzner, P. Pedevilla, A. Zen, and A. Michaelides, "Crystal nucleation in liquids: Open questions and future challenges in molecular dynamics simulations," Chemical Reviews, vol. 116, no. 12, pp. 7078-7116, 2016.

[10] R. Radhakrishnan and B. L. Trout, "Nucleation of hexagonal ice (Ih) in liquid water," J. Am. Chem. Soc., vol. 125 , p. $7743,2003$.

[11] D. Quigley and P. M. Rodger, "Metadynamics simulations of ice nucleation and growth," J. Chem. Phys., vol. 128 , no. 15 , p. $154518,2008$.

[12] P. Geiger and C. Dellago, "Neural networks for local structure detection in polymorphic systems," J. Chem. Phys., vol. 139, no. 16, p. 164105, 2013.

[13] E. B. Moore and V. Molinero, "Is it cubic? ice crystallization from deeply supercooled water," Phys. Chem. Chem. Phys., vol. 13, p. 20008, 2011.

[14] T. L. Malkin, B. J. Murray, A. V. Brukhno, J. Anwar, and C. G. Salzmann, "Structure of ice crystallized from supercooled water," Proceedings of the National Academy of Sciences, vol. 109, no. 4, pp. 1041-1045, 2012.

[15] T. Li, D. Donadio, G. Russo, and G. Galli, "Homogeneous ice nucleation from supercooled water," Phys. Chem. Chem. Phys., vol. 13, pp. 19807-19813, 2011.

[16] A. Haji-Akbari and P. G. Debenedetti, "Direct calculation of ice homogeneous nucleation rate for a molecular model of water," Proceedings of the National Academy of Sciences, vol. 112, no. 34, pp. 10582-10588, 2015.

[17] E. Sanz, C. Vega, J. R. Espinosa, R. Caballero-Bernal, J. L. F. Abascal, and C. Valeriani, "Homogeneous ice nucleation at moderate supercooling from molecular simulation," Journal of the American Chemical Society, vol. 135, no. 40, pp. 15008-15017, 2013.

[18] D. Chakraborty and G. N. Patey, "How crystals nucleate and grow in aqueous nacl solution," The Journal of Physical Chemistry Letters, vol. 4, no. 4, pp. 573-578,
2013.

[19] G. Lanaro and G. N. Patey, "Birth of nacl crystals: Insights from molecular simulations," The Journal of Physical Chemistry B, vol. 120, no. 34, pp. 9076-9087, 2016.

[20] D. Zahn, "Atomistic mechanism of $\mathrm{NaCl}$ nucleation from an aqueous solution," Phys. Rev. Lett., vol. 92, p. 040801, 2004.

[21] N. E. R. Zimmermann, B. Vorselaars, D. Quigley, and B. Peters, "Nucleation of $\mathrm{NaCl}$ from aqueous solution: Critical sizes, ion-attachment kinetics, and rates," Journal of the American Chemical Society, vol. 137, no. 41, pp. 13352-13361, 2015.

[22] M. M. Conde, M. Rovere, and P. Gallo, "Spontaneous nacl-doped ice at seawater conditions: focus on the mechanisms of ion inclusion," Phys. Chem. Chem. Phys., vol. 19, pp. 9566-9574, 2017.

[23] G. Bullock and V. Molinero, "Low-density liquid water is the mother of ice: on the relation between mesostructure, thermodynamics and ice crystallization in solutions," Faraday Discuss., vol. 167, pp. 371-388, 2013.

[24] S. Bauerecker, P. Ulbig, V. Buch, L. Vrbka, and P. Jungwirth, "Monitoring ice nucleation in pure and salty water via high-speed imaging and computer simulations," The Journal of Physical Chemistry C, vol. 112, no. 20, pp. 7631-7636, 2008.

[25] H. Kanno, R. J. Speedy, and C. A. Angell, "Supercooling of water to $-92^{\circ} \mathrm{c}$ under pressure," Science, vol. 189, no. 4206, pp. 880-881, 1975.

[26] J. R. Espinosa, A. Zaragoza, P. Rosales-Pelaez, C. Navarro, C. Valeriani, C. Vega, and E. Sanz, "Interfacial free energy as the key to the pressure-induced deceleration of ice nucleation," Phys. Rev. Lett., vol. 117, p. 135702, 2016.

[27] J. R. Espinosa, G. D. Soria, J. Ramirez, C. Valeriani, C. Vega, and E. Sanz, "Role of salt, pressure, and water activity on homogeneous ice nucleation," J. Phys. Chem. Lett., vol. 8, p. 4486, 2017.

[28] J. L. F. Abascal and C. Vega, "A general purpose model for the condensed phases of water: TIP4P/2005," J. Chem. Phys., vol. 123, p. 234505, 2005.

[29] I. S. Joung and T. E. Cheatham, "Determination of alkali and halide monovalent ion parameters for use in explicitly solvated biomolecular simulations," The Journal of Physical Chemistry B, vol. 112, no. 30, pp. 9020-9041, 2008.

[30] A. L. Benavides, J. L. Aragones, and C. Vega, "Consensus on the solubility of $\mathrm{NaCl}$ in water from computer simulations using the chemical potential route," The Journal of Chemical Physics, vol. 144, p. 124504, 2016.

[31] M. Volmer and A. Weber, "Keimbildung in ubersattigten gebilden," Z. Phys. Chem., vol. 119, p. 277, 1926.

[32] R. Becker and W. Doring, "Kinetische behandlung der keimbildung in ubersattigten dampfen," Ann. Phys., vol. 416, pp. 719-752, 1935.

[33] A. Ladd and L. Woodcock, "Triple-point coexistence properties of the lennard-jones system," Chemical Physics Letters, vol. 51, no. 1, pp. 155 - 159, 1977.

[34] E. Sanz and C. Vega, "Solubility of $\mathrm{KF}$ and $\mathrm{NaCl}$ in water by molecular simulation," J. Chem. Phys., vol. 126, p. $014507,2007$. 
[35] D. Frenkel and B. Smit, Understanding Molecular Simulation. Academic Press, London, 1996.

[36] J. R. Espinosa, C. Vega, C. Valeriani, and E. Sanz, "Seeding approach to crystal nucleation," J. Chem. Phys., vol. 144, p. 034501, 2016.

[37] S. Auer and D. Frenkel, "Prediction of absolute crystalnucleation rate in hard-sphere colloids," Nature, vol. 409, p. 1020, 2001.

[38] J. R. Espinosa, C. Vega, and E. Sanz, "The mold integration method for the calculation of the crystal-fluid interfacial free energy from simulations," J Chem. Phys., vol. 141, no. 13, p. 134709, 2014.

[39] J. R. Espinosa, C. Vega, and E. Sanz, "Ice-water interfacial free energy for the tip4p, tip4p/2005, tip4p/ice and mw models as obtained from the mold integration technique," The Journal of Physical Chemistry C, vol. 120, pp. 8068-8075, 2016.

[40] J. R. Espinosa, E. Sanz, C. Valeriani, and C. Vega, "Homogeneous ice nucleation evaluated for several water models," J. Chem. Phys., vol. 141, p. 18C529, 2014.

[41] H. A. Lorentz, "Ueber die anwendung des satzes vom virial in der kinetischen theorie der gase," Annalen der Physik, vol. 248, no. 1, pp. 127-136, 1881.

[42] D. Berthelot C. R. Acad. Sci., vol. 126, p. 1713, 1898.

[43] W. J. Hamer and Y.-C. Wu, "Osmotic coefficients and mean activity coefficients of uniunivalent electrolytes in water at 25c," Journal of Physical and Chemical Reference Data, vol. 1, no. 4, pp. 1047-1100, 1972.

[44] B. Hess, C. Kutzner, D. van der Spoel, and E. Lindahl, "Algorithms for highly efficient, load-balanced, and scalable molecular simulation," J. Chem. Theory Comput., vol. 4, pp. 435-447, 2008.

[45] M. Parrinello and A. Rahman, "Polymorphic transitions in single crystals: A new Molecular Dynamics method," J. App. Phys., vol. 52, pp. 7182-7190, 1981.

[46] G. Bussi, D. Donadio, and M. Parrinello, "Canonical sampling through velocity rescaling," The Journal of Chemical Physics, vol. 126, no. 1, p. 014101, 2007.

[47] D. R. Wheeler and J. Newman, "A less expensive ewald lattice sum," Chem. Phys. Lett., vol. 366, p. 537, 2002.

[48] B. Hess, H. Bekker, H. J. C. Berendsen, and J. G. E. M. Fraaije, "Lincs: A linear constraint solver for molecular simulations," J. Comput. Chem., vol. 18, no. 12, pp. 1463-1472, 1997.

[49] B. Hess, "P-lincs: a parallel linear constraint solver for molecular simulation," Journal of Chemical Theory and Computation, vol. 4, no. 1, pp. 116-122, 2008.

[50] R. Garcia-Fernandez, J. L. F. Abascal, and C. Vega, "The melting point of ice ih for common water models calculated from direct coexistence of the solid-liquid interface," The Journal of Chemical Physics, vol. 124, no. 14, p. 144506, 2006.

[51] E. G. Noya, C. Vega, and E. de Miguel, "Determination of the melting point of hard spheres from direct coexistence simulation methods," The Journal of Chemical Physics, vol. 128, no. 15, p. 154507, 2008.

[52] J. R. Espinosa, E. Sanz, C. Valeriani, and C. Vega, "On fluid-solid direct coexistence simulations: The pseudohard sphere model," The Journal of Chemical Physics, vol. 139, no. 14, p. 144502, 2013.

[53] J. L. Aragones, E. Sanz, C. Valeriani, and C. Vega, "Calculation of the melting point of alkali halides by means of computer simulations," J. Chem. Phys., vol. 137, no. 10, p. 104507, 2012.
[54] M. M. Conde, M. A. Gonzalez, J. L. F. Abascal, and C. Vega, "Determining the phase diagram of water from direct coexistence simulations: The phase diagram of the TIP4P/2005 model revisited," The Journal of Chemical Physics, vol. 139, no. 15, p. 154505, 2013.

[55] Z. Mester and A. Z. Panagiotopoulos, "Temperaturedependent solubilities and mean ionic activity coefficients of alkali halides in water from molecular dynamics simulations," The Journal of Chemical Physics, vol. 143, no. 4, p. 044505, 2015.

[56] Z. Mester and A. Z. Panagiotopoulos, "Mean ionic activity coefficients in aqueous nacl solutions from molecular dynamics simulations," The Journal of Chemical Physics, vol. 142, no. 4, p. 044507, 2015.

[57] A. S. Paluch, S. Jayaraman, J. K. Shah, and E. J. Maginn, "A method for computing the solubility limit of solids: Application to sodium chloride in water and alcohols," The Journal of Chemical Physics, vol. 133, no. 12, p. $124504,2010$.

[58] F. Moucka, M. Lisal, and W. R. Smith, "Molecular simulation of aqueous electrolyte solubility. 3. alkali-halide salts and their mixtures in water and in hydrochloric acid," The Journal of Physical Chemistry B, vol. 116, no. 18 , pp. 5468-5478, 2012.

[59] I. Nezbeda, F. Moucka, and W. R. Smith, "Recent progress in molecular simulation of aqueous electrolytes: force fields, chemical potentials and solubility," Molecular Physics, vol. 114, no. 11, pp. 1665-1690, 2016.

[60] L. Li, T. Totton, and D. Frenkel, "Computational methodology for solubility prediction: Application to the sparingly soluble solutes," The Journal of Chemical Physics, vol. 146, no. 21, p. 214110, 2017.

[61] R. G. Pereyra, I. Szleifer, and M. A. Carignano, "Temperature dependence of ice critical nucleus size," $J$. Chem. Phys., vol. 135, p. 034508, 2011.

[62] X.-M. Bai and M. Li, "Calculation of solid-liquid interfacial free energy: A classical nucleation theory based approach," J. Chem. Phys., vol. 124, no. 12, p. 124707, 2006.

[63] P. I. Hurtado, J. Marro, and P. L. Garrido, "Demagnetization via nucleation of the nonequilibrium metastable phase in a model of disorder," Journal of Statistical Physics, vol. 133, no. 1, pp. 29-58, 2008.

[64] V. Jacobson, L.C.;Molinero, "Can amorphous nuclei grow crystalline clathrates? the size and crystallinity of critical clathrate nuclei," J.Am.Chem.Soc., vol. 133, p. $6458,2011$.

[65] B. C. Knott, V. Molinero, M. F. Doherty, and B. Peters, "Homogeneous nucleation of methane hydrates: Unrealistic under realistic conditions," J. Am. Chem. Soc., vol. 134, pp. 19544-19547, 2012.

[66] J. R. Espinosa, C. Vega, C. Valeriani, and E. Sanz, "The crystal-fluid interfacial free energy and nucleation rate of $\mathrm{NaCl}$ from different simulation methods," J. Chem. Phys., vol. 142, no. 19, p. 194709, 2015.

[67] A. Zaragoza, M. M. Conde, J. R. Espinosa, C. Valeriani, C. Vega, and E. Sanz, "Competition between ices ih and ic in homogeneous water freezing," The Journal of Chemical Physics, vol. 143, no. 13, p. 134504, 2015.

[68] T. Koop, A. Kapilashrami, L. T. Molina, and M. J. Molina, "Phase transitions of sea-salt/water mixtures at low temperatures: Implications for ozone chemistry in the polar marine boundary layer," Journal of Geophysical Research: Atmospheres, vol. 105, no. D21, pp. 26393- 
$26402,2000$.

[69] T. Koop, B. Luo, A. Tsias, and T. Peter, "Water activity as the determinant for homogeneous ice nucleation in aqueous solutions," Nature, vol. 406, pp. 611-614, 2000.

[70] H. R. Pruppacher, "A new look at homogeneous ice nucleation in supercooled water drops," J. Atmosph. Sci., vol. 52, p. 1924, 1995.

[71] B. J. Murray, S. L. Broadley, T. W. Wilson, S. J. Bull, R. H. Wills, H. K. Christenson, and E. J. Murray, "Kinetics of the homogeneous freezing of water," Phys. Chem. Chem. Phys., vol. 12, p. 10380, 2010.
[72] A. Manka, H. Pathak, S. Tanimura, J. Wolk, R. Strey, and B. E. Wyslouzil, "Freezing water in no man's land," Phys. Chem. Chem. Phys., vol. 14, pp. 4505-4516, 2012.

[73] R. W. Potter, M. A. Clynne, and D. L. Brown, "Freezing point depression of aqueous sodium chloride solutions," Scientific Communications, vol. 73, no. 2, pp. 284-285, 1978.

[74] W. Lechner and C. Dellago, "Accurate determination of crystal structures based on averaged local bond order parameters," J. Chem. Phys., vol. 129, no. 11, p. 114707, 2008. 